\title{
Control of the transient subdiffusion exponent at short and long times
}

\author{
Aleksander Stanislavsky (1)* \\ Institute of Radio Astronomy, 4 Mystetstv Street, 61002 Kharkiv, Ukraine \\ Aleksander Weron ${ }^{\dagger}$ \\ Faculty of Pure and Applied Mathematics, Hugo Steinhaus Center, Wroctaw University of Science and Technology, \\ Wybrzeże Wyspiańskiego 27, 50-370 Wroctaw, Poland
}

(Received 18 May 2019; published 6 September 2019)

\begin{abstract}
We consider a simple, physically and biologically motivated model of transient subdiffusion having different exponents in the mean squared displacement at short and long times. For this purpose, the sum of two independent stable processes (with different indexes) as subordinators is used. Based on their infinitely divisible properties, the probability density function of particle displacements and the Fokker-Planck-type equation are obtained. Manifesting different origins of inhomogeneities (traps) in the system, the subordinators' indexes affect the asymptotic behavior of the mean squared displacement for a particle walking in the environment. Links to the Jonscher universal relaxation law and the Prabhakar-type memory functions are given.
\end{abstract}

DOI: 10.1103/PhysRevResearch.1.023006

\section{INTRODUCTION}

Normal diffusion motions are often observed in homogeneous media, from colloidal and dilute suspensions, emulsions, and simple polymeric solutions to many biological systems. In living cells, the diffusion transport plays an important role and occurs in complex and crowded intracellular environments. This leads to major changes of the motion (see Ref. [1] and references therein). In freely diffusing molecules and tracers, the mean squared displacement (MSD) increases linearly in time, whereas in living cells, due to the complex environment, the MSD demonstrates nonlinear growth, typically slower than for normal diffusion. The pure subdiffusion manifests the MSD, proportional to a power law $\operatorname{MSD}(t) \propto t^{\alpha}$, with an exponent $0<\alpha \leqslant 1$. The behavior is explained by the dominating influence of traps so that the waiting times of random walks become $\alpha$ stable with an infinite mean. Generally, the origins of anomalous diffusion can be a variety: disorder in heterogeneous media, random traps, barriers or comblike structures in materials, transport processes occurring in complex setups, etc. Their lifetimes can be different. It is no wonder that the complex natural systems show transitions between diffusive regimes, each of which has a MSD trend itself. Then the transient diffusion possesses the exponent $\alpha$ changing in time. The exponent is a fingerprint of anomalous subdiffusion, characterizing, for example, crowding concentration [2], the impact on which

\footnotetext{
*a.a.stanislavsky@ rian.kharkov.ua

†aleksander.weron@pwr.edu.pl

Published by the American Physical Society under the terms of the Creative Commons Attribution 4.0 International license. Further distribution of this work must maintain attribution to the author(s) and the published article's title, journal citation, and DOI.
}

makes it possible to influence the processes in the cells [3]. In general, it is not simple to identify the mechanisms underlying transient anomalous diffusion, as each diffusion within complex media is hindered by many factors. This motivates development of various methods for their study [4-7]. The analysis of different scenarios of anomalous diffusion will be more creative if the description of such systems are reduced to a composition of simpler and more understandable diffusion systems. A benefit of using well-defined model systems is the opportunity to perform a detailed statistical analysis of anomalous transport at a given point in parameter space. The stochastic representation for a wider class of the anomalous diffusion phenomena with a more arbitrary nonlinear MSD and their identification is of great interest [8,9]. The present work aims at shedding light on diffusion in complex media in which many disorder mechanisms are simultaneously present.

\section{SIMPLE POWER-LAW KERNEL}

The subdiffusive dynamics is excellently written as a rescaled diffusive motion $X(\tau)$ subordinated by an inverse Lévy-stable random process $S_{\alpha}(t)$, independent of the former [10]. In this case, the stochastic process $X(\tau)$ has the probability density function (PDF) $h(x, \tau)$ satisfying the ordinary Fokker-Planck (FP) equation

$$
\partial h(x, \tau) / \partial \tau=\hat{L}(x) h(x, \tau),
$$

where $\hat{L}(x)$ is the time-independent FP operator (for example, $-\frac{\partial}{\partial x} F(x)+D \frac{\partial^{2}}{\partial x^{2}}$ with a force $\left.F\right)$. In a general case, the operator $\hat{L}$ can be both multidimensional and fractional in space $[11,12]$. The operational time $\tau=S_{\alpha}(t)$ is obtained from a strictly increasing $\alpha$-stable Lévy process $T_{\alpha}(\tau)$ which is nothing else but the continuous limit of a sequence $T_{i}$, $i=1,2, \ldots$ of non-negative, independent, identically distributed random variables (obeying an $\alpha$-stable Lévy PDF) 
representing waiting-time intervals between subsequent jumps of a walker [13]. If $g_{\alpha}(t, \tau)$ is the PDF of $T_{\alpha}(\tau)$, the mean $\left\langle e^{-u T_{\alpha}(\tau)}\right\rangle$ is the Laplace transform of $g_{\alpha}(t, \tau)$ equal to

$$
\left\langle e^{-u T_{\alpha}(\tau)}\right\rangle=\int_{0}^{\infty} e^{-u t} g_{\alpha}(t, \tau) d t=e^{-\tau u^{\alpha}}, \quad 0<\alpha<1 .
$$

Then the operational time $S_{\alpha}(t)$ as an inverse process to $T(\tau)$ fulfills the following relation:

$$
S_{\alpha}(t)=\inf \left\{\tau>0: T_{\alpha}(\tau)>t\right\} .
$$

Denoting the PDF of the operational time $S(t)$ as $f(\tau, t)$, its Laplace transform takes the simple form

$$
\tilde{f}(\tau, s)=s^{\alpha-1} e^{-\tau s^{\alpha}} .
$$

Using it, it is not difficult to obtain the fractional FokkerPlanck (FP) equation

$$
p_{\alpha}(x, t)=q(x)+\frac{1}{\Gamma(\alpha)} \int_{0}^{t} d \tau(t-\tau)^{\alpha-1} \hat{L}(x) p_{\alpha}(x, \tau)
$$

for describing the subdiffusion [14]. The interpretation of this behavior is enough simple: The motion of a walker meets random traps generating waiting-time intervals with $\alpha$-stable Lévy properties between subsequent jumps of the walker.

\section{TRANSIENT DIFFUSION}

The right-skewed $\alpha$-stable distribution is a particular case of infinitely divisible distributions. Among them, exponential, $\gamma$, Gaussian, inverse Gaussian, and others are well known. Because of the Lévy-Khintchine formula [15], they are characterized by the exponentially weighted function

$$
\left\langle e^{-u T_{\Psi}(\tau)}\right\rangle=e^{-\tau \Psi(u)}=\int_{0}^{\infty} e^{-u t} g_{\Psi}(t, \tau) d t,
$$

where $\Psi(u)$ is called the Laplace exponent and $g_{\Psi}(t, \tau)$ is the PDF of this process. As it has been shown [16-19], the subordination of $X(\tau)$ by $S_{\Psi}(t)=\inf \left\{\tau>0: T_{\Psi}(\tau)>t\right\}$ changes a character of both MSD and the FP equation form. Particularly, the FP equation has a general form

$$
p(x, t)=q(x)+\int_{0}^{t} d \tau M(t-\tau) \hat{L}(x) p(x, \tau),
$$

where $M(t)$ is the memory function [20]. The kernel $M(t)$ can be represented as an inverse Laplace transform $L_{t}^{-1}$

$$
M(t)=\frac{1}{2 \pi i} \int_{c-i \infty}^{c+i \infty} \frac{e^{s t}}{\Psi(s)} d s=L_{t}^{-1} \frac{1}{\Psi(s)},
$$

where $c$ is large enough that $1 / \Psi(s)$ is defined for $\operatorname{Re} s \geqslant c$, and $i^{2}=-1$. The PDF of the operational time $S_{\Psi}(t)$ is simply written as a Laplace image,

$$
\tilde{f}(\tau, s)=\frac{\Psi(s)}{s} e^{-\tau \Psi(s)} .
$$

Then the solution (propagator) of Eq. (7) takes the form of a subordination integral,

$$
p(x, t)=\int_{0}^{\infty} h(x, \tau) f(\tau, t) d \tau .
$$

Infinitely divisible distributions describe a wide class of random processes. If, as an example, the tempered $\alpha$-stable subordinator (having all moments of operational time) is taken [21], then the diffusive picture occupies an intermediate place between subdiffusion and normal diffusion. In this case, the Laplace exponent is $\Psi(s)=(s+\delta)^{\alpha}-\delta^{\alpha}$, where $\delta$ is a positive constant and $0<\alpha<1$. If $\delta$ tends to zero, the tempered $\alpha$-stable process becomes simply $\alpha$ stable. The memory function has an analytical form, namely $M(t)=$ $e^{-\delta t} t^{\alpha-1} E_{\alpha, \alpha}\left(\delta^{\alpha} t^{\alpha}\right)$, expressed in terms of the two-parameter Mittag-Leffler function $E_{\alpha, \beta}(z)=\sum_{k=0}^{\infty} z^{k} / \Gamma(\alpha k+\beta)$ with $\alpha>0$ and $\beta>0$. For $t \ll 1$ (or $\delta \rightarrow 0$ ), this memory function leads to $t^{\alpha} / \Gamma(\alpha)$ as the kernel in Eq. (5), whereas for $t \gg 1$ (or $\alpha \rightarrow 1$ ) it becomes constant and, as a result, Eq. (7) transforms into the ordinary FP equation.

\section{STOCHASTIC REPRESENTATION OF ANOMALOUS DIFFUSION WITH A MULTIPLE SUBORDINATION}

According to the well-known handbook of Feller [15], the function $w$ is the Laplace transform of an infinitely divisible probability distribution if and only if $w=e^{-\psi}$, where $\psi$ is a Bernstein function and $\psi\left(0_{ \pm}\right)=0$. Each Bernstein function is non-negative, and its first derivative is a completely monotonic (CM) function. Moreover, for a Bernstein function, its reciprocal is $\mathrm{CM}$ [22]. It should be noticed that the sum of Bernstein functions $\psi_{1}+\psi_{2}$, as well their composition $\psi_{1}$ 。 $\psi_{2}$, is also a Bernstein function. This allows us to create new infinitely divisible processes from known ones. The procedure covers an arbitrary number of compositions and sum terms. Without restricting the generality, we study the operations on two infinitely divisible processes.

Consider two independent infinitely divisible random processes $T_{1}$ and $T_{2}$ with the Laplace exponents $\psi_{1}$ and $\psi_{2}$, respectively. Then the exponentially weighted function of their sum is

$$
\left\langle e^{-u\left[T_{1}(\tau)+T_{2}(\tau)\right]}\right\rangle=e^{-\tau\left(\psi_{1}(u)+\psi_{2}(u)\right)} .
$$

The inverse process of the sum has the PDF with the Laplace image

$$
\tilde{f}_{\Sigma}(\tau, s)=\frac{\left[\psi_{1}(s)+\psi_{2}(s)\right]}{s} e^{-\tau\left(\psi_{1}(s)+\psi_{2}(s)\right)} .
$$

If we go to the composition of two processes $T_{1}$ and $T_{2}$, then the exponentially weighted function of the resulting process takes the form

$$
\left\langle e^{-u T_{1}\left(T_{2}(\tau)\right)}\right\rangle=e^{-\tau \psi_{1}\left(\psi_{2}(u)\right)} .
$$

Using the process as a governing one for a diffusion motion $X(\tau)$, the composition leads to a double subordination $X\left[S_{1}\left(S_{2}(t)\right)\right]$ satisfying the following integral relation,

$$
p(x, t)=\int_{0}^{\infty} \int_{0}^{\infty} h\left(x, \tau_{1}\right) f_{1}\left(\tau_{1}, \tau_{2}\right) f_{2}\left(\tau_{2}, t\right) d \tau_{1} d \tau_{2},
$$

where $f_{1}$ and $f_{2}$ are the PDFs of the inverse processes $S_{1}(t)$ and $S_{2}(t)$, respectively. Note that the process $S_{1}\left(S_{2}(t)\right)$ jumps at the occurrence of events of the inner process $S_{2}(t)$. This allows one to inset one random process into another as a random time [23]. The Laplace image of the PDF of the 
composition $S_{1}\left(S_{2}(t)\right)$ yields

$$
\begin{aligned}
\tilde{f}_{\circ}(\tau, s) & =\mathcal{L}_{t}\left(f_{\circ}(\tau, t)\right) \\
& =\int_{0}^{\infty} f_{1}(\tau, r) \mathcal{L}_{t}\left(f_{2}(r, t)\right) d r \\
& =\int_{0}^{\infty} f_{1}(\tau, r) \frac{\psi_{2}(s)}{s} e^{-r \psi_{2}(s)} d r \\
& =\frac{\psi_{2}(s)}{s} \int_{0}^{\infty} f_{1}(\tau, r) e^{-r \psi_{2}(s)} d r \\
& =\frac{\psi_{2}(s)}{s} \frac{\psi_{1}\left(\psi_{2}(s)\right)}{\psi_{2}(s)} e^{-\tau \psi_{1}\left(\psi_{2}(s)\right)} \\
& =\frac{\psi_{1}\left(\psi_{2}(s)\right)}{s} e^{-\tau \psi_{1}\left(\psi_{2}(s)\right)} .
\end{aligned}
$$

The generalization to more general cases with many compositions and sum terms is not difficult to derive.

\section{SUM OF $\alpha$-STABLE SUBORDINATORS}

One case deserves special attention. If $\psi_{1}(u)=u^{\alpha}$ and $\psi_{2}(u)=u^{\beta}$, then $\psi_{1}\left(\psi_{2}(u)\right)=u^{\alpha \beta}$. This means that a stable $\alpha$ process subordinated by a stable $\beta$ process leads to a stable $\alpha \beta$ process [15]. Then the composition of two independent inverse stable subordinators $S_{\alpha}\left(S_{\beta}(t)\right)$ with the PDF $f_{\circ}(\tau, t)$ satisfies a subordination integral

$$
f_{\circ}(\tau, t)=\int_{0}^{\infty} f_{\alpha}(\tau, r) f_{\beta}(r, t) d r .
$$

After the Laplace transform, we obtain the PDF image of the composition in the form $\tilde{f}_{\circ}(\tau, s)=s^{\alpha \beta-1} e^{-\tau s^{\alpha \beta}}$ as expected [24]. Thus, this process is the same in distribution as the inverse stable subordinator of index $\alpha \beta$. It may seem that the composition of two power-law distributions gives a relatively uninteresting result, but other choices of processes may give more interesting results (see Sec. VI as an example).

If we come to a sum of two independent stable subordinators with different indexes [25], $\alpha$ and $\beta$, then $\psi_{1}(u)+$ $\psi_{2}(u)=C_{1} u^{\alpha}+C_{2} u^{\beta}$ for $C_{1}+C_{2}=1$. In this case, the memory function has the Laplace image $\tilde{M}(s)=1 /\left(C_{1} s^{\alpha}+C_{2} s^{\beta}\right)$ with $\alpha>\beta$. After the inverse Laplace transform, it takes the following form:

$$
M(t)=\frac{1}{C_{1}} t^{\alpha-1} E_{\alpha-\beta, \alpha}\left(-C_{2} t^{\alpha-\beta} / C_{1}\right) .
$$

Knowing the memory function, it is not difficult to derive the corresponding FP equation. It is interesting that in this case we can write it in the form of fractional derivatives [26], namely

$$
C_{1} \frac{\partial^{\alpha} p_{\alpha, \beta}(x, t)}{\partial t^{\alpha}}+C_{2} \frac{\partial^{\beta} p_{\alpha, \beta}(x, t)}{\partial t^{\beta}}=\hat{L}(x) p_{\alpha, \beta}(x, t),
$$

where $\partial^{\alpha} / \partial t^{\alpha}$ and $\partial^{\beta} / \partial t^{\beta}$ is the Caputo derivative. The sum of stable subordinators leads to the model of retarding diffusion [27]. Really, if the moments of a parent process $X(\tau)$ are known, as in the case of the Brownian motion, the moments of the process $X\left[S_{\Phi}(t)\right]$ [here the index $\Phi$ indicates the Laplace exponent, for example, $\Phi(s)=\psi_{1}\left(\psi_{2}(s)\right)$ or $\Phi(s)=\psi_{1}(s)+\psi_{2}(s)$ in the simplest case] subordinated by a multiple combination (sum and/or composition) of directing

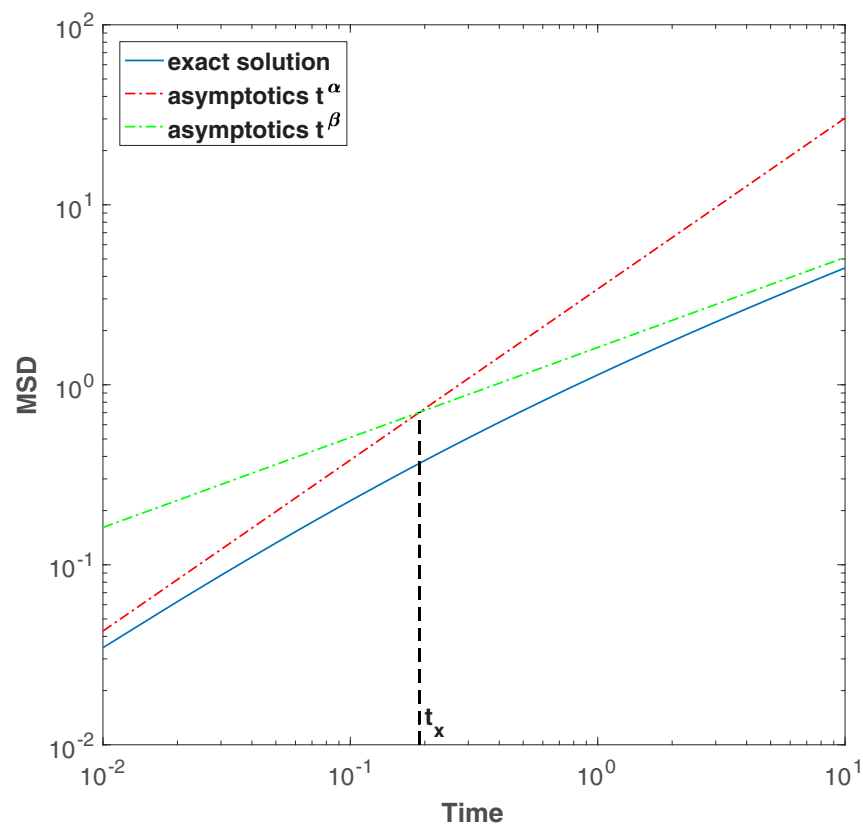

FIG. 1. Mean squared displacement of anomalous diffusion (for $D=1$ ) with the memory function (17) under $\alpha=0.95, \beta=0.5$, $C_{1}=0.3$, and $C_{2}=0.7$. The notation $t_{\mathrm{x}}$ shows the crossover time.

random processes can be found by analytical calculations. Using the second moment of the Brownian motion in the form $\left\langle B^{2}(\tau)\right\rangle=D \tau$, where $D$ is a diffusive constant, the meansquared displacement of $Y(t)=B\left[S_{\Phi}(t)\right]$ reads

$$
\left\langle B^{2}\left[S_{\Phi}(t)\right]\right\rangle=D \int_{0}^{t} M(y) d y .
$$

The MSD of the diffusion process Eq. (17) with $D=1$ is equal to

$$
\left\langle x^{2}\right\rangle=\frac{1}{C_{1}} t^{\alpha} E_{\alpha-\beta, \alpha+1}\left(-C_{2} t^{\alpha-\beta} / C_{1}\right),
$$

having the following asymptotic behavior: $\lim _{t \rightarrow 0}\left\langle x^{2}\right\rangle=$ $\frac{1}{C_{1}} t^{\alpha} / \Gamma(\alpha+1)$ and $\lim _{t \rightarrow \infty}\left\langle x^{2}\right\rangle=\frac{1}{C_{2}} t^{\beta} / \Gamma(\beta+1)$. As $\alpha>\beta$, the transient diffusion is retarding (Fig. 1). The interpretation of this behavior is quite simple: The motion of a walker meets two types of different random traps generating waiting-time intervals with stable Lévy properties, having two indexes $\alpha$ and $\beta$, between subsequent jumps of the walker. As a first approximation, using the asymptotic values, it is easy enough to find the crossover time $t_{\mathrm{x}}=\left[\left(C_{1} \Gamma(\alpha+1)\right) /\left(C_{2} \Gamma(\beta+1)\right)\right]^{1 /(\alpha-\beta)}$, shown in Fig. 1.

Taking the Brownian motion as a parent process, the Laplace image $\tilde{p}(x, s)$ is written as the tabulated integral [28], expressed in terms of the modified Bessel function of the third kind. Fortunately, its index is equal to $1 / 2$. Thus, we find

$$
\tilde{p}(x, s)=\frac{1}{\sqrt{2 D}} \frac{\sqrt{\Phi(s)}}{s} \exp \left(-2 \frac{|x|}{\sqrt{2 D}} \sqrt{\Phi(s)}\right) .
$$

Using numerical methods, the sum of two stable subordinators, directing the Brownian motion, gives a propagator shown in Fig. 2. 


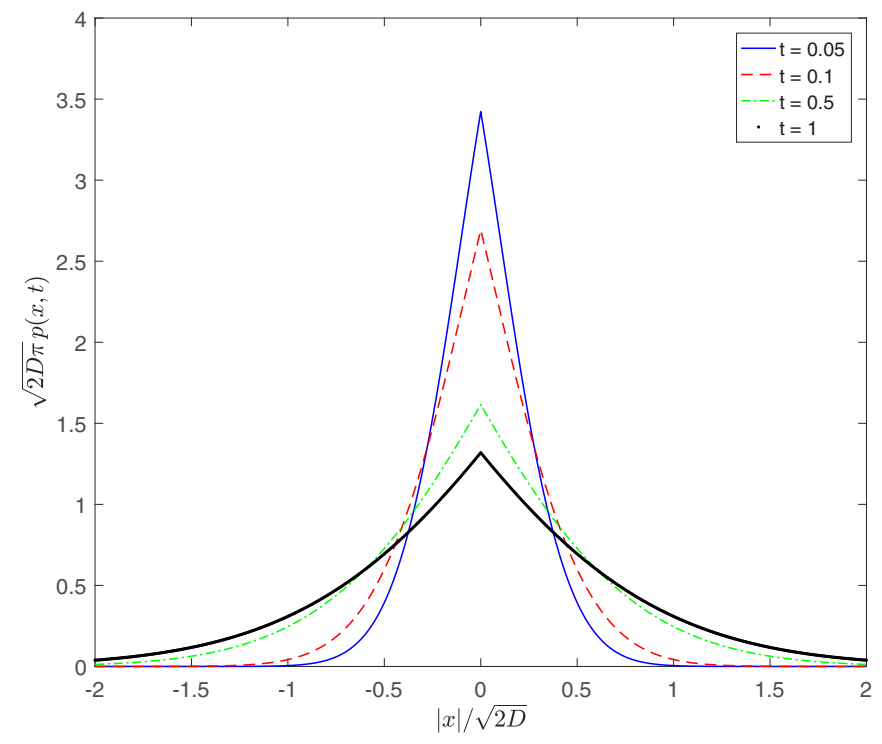

FIG. 2. Propagator $p(x, t)$ for the anomalous diffusion with a constant potential under the memory function (17) with $\alpha=0.95$ and $\beta=0.5$, drawn for consecutive dimensionless instances of time. Starting as the Dirac $\delta$ function, the value $p(x, t)$ becomes infinitesimal for $t \rightarrow \infty$.

\section{PRABHAKAR-TYPE MEMORY FUNCTION}

If the memory function of Eq. (7) is described by the following expression,

$$
M(t)=t^{\mu-1} E_{\rho, \mu}^{\gamma}\left(-\lambda t^{\rho}\right),
$$

then the FP equation is expressed in terms of the Prabhakar integral operator [29,30]. Here the Prabhakar-type kernel is expressed in terms of the three-parameter MittagLeffler function, having the following Taylor series $E_{\alpha, \beta}^{\rho}(x)=$ $\sum_{i=0}^{\infty} \frac{(\rho, i) x^{i}}{\Gamma(\alpha i+\beta) i !}$ with $\alpha, \beta>0$, where $(\rho, i)=\rho(\rho+1)(\rho+$ $2) \ldots(\rho+i-1)$ is the Appell's symbol with $(\rho, 0)=1, \rho \neq$ 0 . If $\gamma=0$, then $E_{\rho, \mu}^{0}(x)=1 / \Gamma(\mu)$, and the Prabhakar operator transforms into the Riemann-Liouville fractional integral of order $\mu$, generalizing the classical fractional calculus [31]. By the Laplace tabulated formula

$$
t^{\mu-1} E_{\rho, \mu}^{\gamma}\left(a t^{\rho}\right)=L_{t}^{-1}\left[s^{-\mu}\left(1-a s^{-\rho}\right)^{-\gamma}\right],
$$

we have the corresponding Laplace exponent $s^{\mu}\left(1+\lambda s^{-\rho}\right)^{\gamma}$, where $\lambda \geqslant 0$ is a constant. This function can be represented as $\left[s^{\mu / \gamma}+\lambda s^{\mu / \gamma-\rho}\right]^{\gamma}$. Then the diffusive process $X(\tau)$, subordinated by such an inverse process, can be presented as a double subordination, consisting of a sum of two independent stable processes composed with a third independent inverse stable process, i.e., $X\left[S_{\gamma}\left(S_{\mu / \gamma, \mu / \gamma-\rho}(t)\right)\right]$. Using Eq. (22) and the Brownian motion, as a parent process, the MSD of the transient anomalous diffusion is written in an explicit form $\left\langle B^{2}[S(t)]\right\rangle=D t^{\mu} E_{\rho, \mu+1}^{\gamma}\left(\lambda t^{\rho}\right)$. On the one hand, the MSD behaves as $\lim _{t \rightarrow 0} t^{\mu} E_{\rho, \mu+1}^{\gamma}\left(\lambda t^{\rho}\right)=t^{\mu} / \Gamma(\mu+1)$, whereas for large $t$ it tends to $\lim _{t \rightarrow \infty} t^{\mu} E_{\rho, \mu+1}^{\gamma}\left(-\lambda t^{\rho}\right)=$ $\lambda^{-\gamma} t^{\mu-\rho \gamma} / \Gamma(\mu-\rho \gamma+1)$. If $\gamma=1$, the third subordinator becomes degenerate. Consequently, we come to a simpler model, the sum of two stable subordinators, considered above, and have also a crossover between two subdiffusion exponents. Thus, the complex dynamics involving many stable subordinators describes anomalous diffusion in disorder media with heterogeneities, characterizing their multiscale behavior.

\section{NUMERICAL APPROXIMATION OF SAMPLE PATHS}

Let us now show how to get sample paths of the anomalous diffusion process with the memory function (17), i.e., $Y(t)=$ $X\left(S_{\alpha, \beta}(t)\right)$. Our simulations use explicitly the fact that $Y(t)$ is a subordinated process. Every sample path of $Y(t)$ is considered as a superposition of independently generated trajectories of $X(\tau)$ and $S_{\alpha, \beta}(t)$. If we want to simulate $Y(t)$ on the interval $[0, T]$, where $T$ is the time horizon, the proposed algorithm includes the following steps:

(1) Each trajectory of the inverse process $S_{\alpha, \beta}(t)$ is approximated by the process $S_{\alpha, \beta, \Delta t}(t)=[\min \{n \in \mathbb{N}$ : $\left.\left.C_{1} T_{\alpha}(n \Delta t)+C_{2} T_{\beta}(n \Delta t)>t\right\}-1\right] \Delta t$, where $t$ is the step length, and $t \in[0, T]$. Insofar as $\sup _{t \in[0, T]} \mid S_{\alpha, \beta, \Delta t}(t)-$ $S_{\alpha, \beta}(t) \mid \leqslant \Delta t$, as smaller $\Delta t$ is chosen, the approximation improves. To reproduce numerically the process $S_{\alpha, \beta, \Delta t}(t)$, we generate $T_{\alpha}(n \Delta t)$ and $T_{\beta}(n \Delta t)$ for $n=1,2, \ldots$ Using the standard method of summing increments of the Lévy process $T_{\alpha}(t)$, we get $T_{\alpha}(0)=0, T_{\alpha}(n \Delta t)=T_{\alpha}([n-1] \Delta t)+$ $(\Delta t)^{1 / \alpha} \eta_{k}$, where $\eta_{k}$ are the independent totally skewed positive $\alpha$-stable random variables generated by the well-known method [32]. The second independent process $T_{\beta}(t)$ is simulated similarly.

(2) The process $X(\tau)$ is given by the Itô stochastic differential equation, namely $d X(\tau)=F(X(\tau)) d \tau+d B(\tau)$, where $B(\tau)$ is the ordinary Brownian motion, mentioned above. To approximate it, we use the classical Euler scheme [33], i.e., $X(0)=0, \quad X\left(\tau_{k}\right)=X\left(\tau_{k-1}\right)+F\left(X\left(\tau_{k-1}\right)\right) \Delta t+$ $\Delta t^{1 / 2} \xi_{k}$, where $\xi_{k}$ are independent random variables having the standard normal distribution, and $k=1,2, \ldots, N$.

(3) Finally, putting the approximated trajectories of $S_{\alpha, \beta}(t)$ and $X(\tau)$ together, we obtain the trajectory of the anomalous diffusion $X\left(S_{\alpha, \beta}(t)\right)$ for arbitrary external forces.

In Fig. 3, we show typical trajectories of the inverse subordinator and anomalous subdiffusion with the memory function (17) in the force-free case.

\section{NONEXPONENTIAL RELAXATION}

The subordination point of view becomes very popular in the study of relaxation law types, as this approach permits us to consider the relaxation as a form of the anomalous diffusion [34]. In this framework, the relaxation function $\phi(t)$ relates to the temporal decay of a given mode $k$ that can be expressed through the Fourier transform of the diffusion process $X\left[S_{\Phi}(t)\right]$, namely

$$
\phi(t)=\left\langle e^{i k X\left[S_{\Phi}(t)\right]}\right\rangle,
$$

where $k>0$ has the physical meaning of a wave number (the Fourier image of spatial coordinates). In the frequency domain of measurements, it is more convenient to use the shape function, defined as a Fourier transform of the relaxation response $f(t)=-\phi(t) / d t$, i.e.,

$$
\phi^{*}(\omega)=\int_{0}^{\infty} e^{-i \omega t}\left(-\frac{d \phi(t)}{d t}\right) d t .
$$



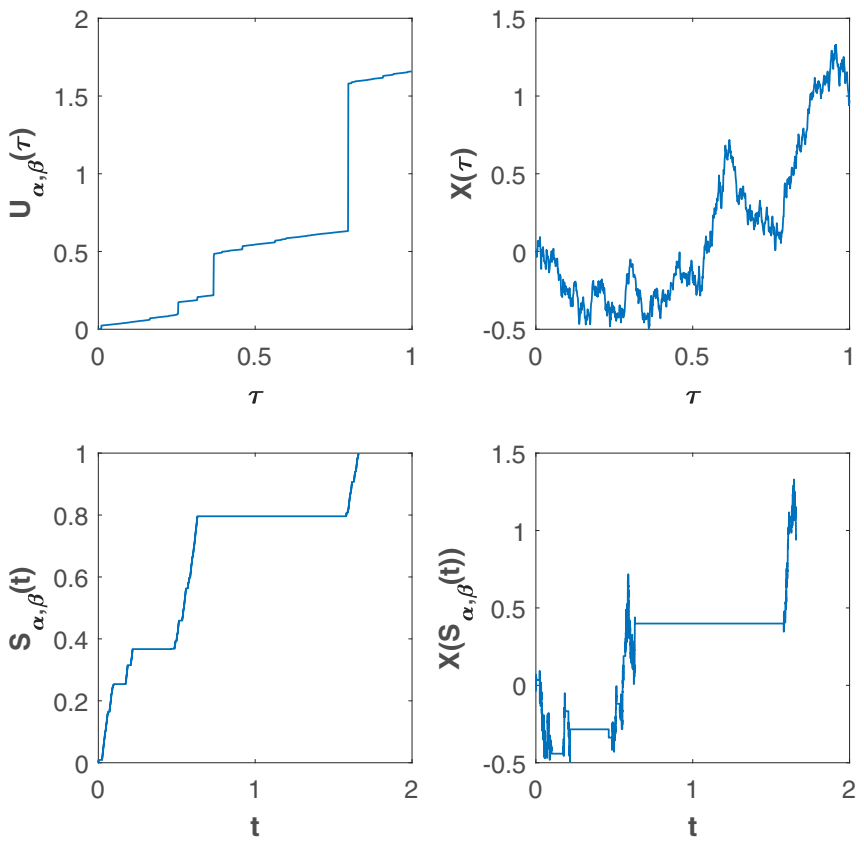

FIG. 3. An example of trajectories for the anomalous diffusion with a constant potential under the memory function (17) with $\alpha=$ 0.95 and $\beta=0.5$, where $U_{\alpha, \beta}(\tau)=C_{1} T_{\alpha}(\tau)+C_{2} T_{\beta}(\tau)$.

In a general case, the above frequency-domain relaxation function takes the following form,

$$
\phi^{*}(\omega)=\frac{1}{1+\Phi(i \omega) / \Xi(k)},
$$

where $\Xi(k)$ denotes the logarithm of the characteristic function of the process $X(\tau)$ [19].

As applied to the memory function Eq. (17), the frequencydomain relaxation function reads

$$
\phi^{*}(\omega)=\frac{1}{1+C_{1}\left(i \omega / \omega_{p}\right)^{\alpha}+A C_{2}\left(i \omega / \omega_{p}\right)^{\beta}},
$$

where $\omega_{p}>0$ is the normalizing frequency constant (or the loss peak frequency characteristic for an investigated material) and $A$ is a model constant. Based on the approach, developed above, we can propose the interpretation of this relaxation law. Recall that each relaxation law exhibits the fractional-power dependence in the complex dielectric susceptibility $\chi(\omega)=$ $\chi^{\prime}(\omega)-i \chi^{\prime \prime}(\omega)$. As was well known [35], the complex dielectric susceptibility can be represented as $\chi(\omega)=$ $\phi^{*}(\omega)\left(\chi_{0}-\chi_{\infty}\right)+\chi_{\infty}$. The constant $\chi_{\infty}$ represents the asymptotic value of the susceptibility $\chi(\omega)$ at high frequencies, and $\chi_{0}$ characterizes the opposite limit. In the case of the law (27), we obtain $\chi(\omega) \propto\left(i \omega / \omega_{p}\right)^{-\alpha}$ for $\omega \gg \omega_{p}$ and $\Delta \chi(\omega) \propto\left(i \omega / \omega_{p}\right)^{\beta}$ for $\omega \ll \omega_{p}$, where $\Delta \chi(\omega)=\chi_{0}-$ $\chi(\omega)$. Thus, the relaxation pattern relates to two-power laws of relaxation. Particularly, the celebrated Havriliak-Negami (HN) law has similar two-power behavior of relaxation. The exponents $n=1-\alpha$ and $m=\beta$ fall in the range $(0,1)$. As is well known from the book of Jonscher [35], for majority of the relaxation data the exponents fulfill the relation $m \geqslant 1-n$ while for rest of the data satisfy the opposite one: $m<1-n$. Note that the frequency-domain relaxation function (27) may describe the relaxation data with $m \leqslant 1-n$.

\section{CONCLUSIONS}

A main feature of the transient anomalous diffusion is the presence of many different disorder mechanisms leading to a strong heterogeneity in the medium through which a walker wanders. The simplest model of anomalous diffusion, characterized by the two-scaled MSD of a walking particle, arises from the sum of two independent stable processes as subordinators. In the case of the Prabhakar-type memory function, the diffusion is a result of a double subordination, in which the sum of two independent stable subordinators directs the third independent inverse stable. A more general approach suggests using an arbitrary number of compositions and sum terms. If the character of the memory function evolution in time for a particular diffusion was found, one can restore the Laplace exponent of the subordinator responsible for such a diffusion process, establish its properties (particularly, deciding whether the given subordinator is decomposable or not), and ultimately penetrate the essence of observed complex natural phenomena, moving from a wide variety of FP equations with memory (macro level) to their description in the language of subordinated random processes (micro and/or meso levels).

\section{ACKNOWLEDGMENTS}

A.S. is grateful to the Hugo Steinhaus Center for pleasant hospitality during his visit in Wrocław University of Science and Technology. This research was partially supported by DFG-NCN Beethoven Grant No. DFG-NCN 2016/23/G/ST1/04083.
[1] F. Höfling and T. Franosch, Anomalous transport in the crowded world of biological cells, Rep. Prog. Phys. 76, 046602 (2013).

[2] D. S. Banks and C. Fradin, Anomalous diffusion of proteins due to molecular crowding, Biophys. J. 89, 2960 (2005).

[3] T. Sungkaworn, M.-L. Jobin, K. Burnecki, A. Weron, M. J. Lohse, and D. Calebiro, Single-molecule imaging reveals receptor-G protein interactions at cell surface hot spots, Nature (London) 550, 543 (2017).
[4] K. Weron, A. Jurlewicz, M. Magdziarz, A. Weron, and J. Trzmiel, Overshooting and undershooting subordination scenario for fractional two-power-law relaxation responses, Phys. Rev. E 81, 041123 (2010).

[5] P. Siegle, I. Goychuk, and P. Hänggi, Markovian embedding of fractional superdiffusion, Europhys. Lett. 93, 20002 (2011).

[6] I. Goychuk, Viscoelastic subdiffusion: Generalized Langevin equation approach, Adv. Chem. Phys. 150, 187 (2012).

[7] D. Molina-Garcia, T. Sandev, H. Safdari, G. Pagnini, A. Chechkin, and R. Metzler, Crossover from anomalous to normal 
diffusion: Truncated power-law noise correlations and applications to dynamics in lipid bilayers, New J. Phys. 20, 103027 (2018).

[8] Y. Lanoiselée and D. S. Grebenkov, Non-Gaussian diffusion of mixed origins, J. Phys. A: Math. Theor. 52, 304001 (2019).

[9] S. Carnaffan and R. Kawai, Analytic model for transient anomalous diffusion with highly persistent correlations, Phys. Rev. E 99, 062120 (2019).

[10] A. Weron and M. Magdziarz, Anomalous diffusion and semimartingales, Europhys. Lett. 86, 60010 (2009).

[11] E. Barkai, R. Metzler, and J. Klafter, From continuous time random walks to the fractional Fokker-Planck equation, Phys. Rev. E 61, 132 (2000).

[12] G. Baumann and F. Stenger, Fractional Fokker-Planck equation, Mathematics 5, 12 (2017).

[13] M. M. Meerschaert and H. P. Scheffler, Limit theorems for continuous-time random walks with infinite mean waiting times, J. Appl. Probab. 41, 623 (2004).

[14] R. Metzler, E. Barkai, and J. Klafter, Anomalous Diffusion and Relaxation Close to Thermal Equilibrium: A Fractional Fokker-Planck Equation Approach, Phys. Rev. Lett. 82, 3563 (1999).

[15] W. Feller, Introduction to Probability Theory and Its Application (John Wiley \& Sons, New York, 1967), Vol. II.

[16] J. Janczura and A. Wyłomańska, Anomalous diffusion models: Different types of subordinator distribution, Acta Phys. Polon. B 43, 1001 (2012).

[17] S. Orzeł, W. Mydlarczyk, and A. Jurlewicz, Accelerating subdiffusions governed by multiple-order time-fractional diffusion equations: Stochastic representation by a subordinated Brownian motion and computer simulations, Phys. Rev. E 87, 032110 (2013).

[18] A. Stanislavsky, K. Weron, and A. Weron, Anomalous diffusion with transient subordinators: A link to compound relaxation laws, J. Chem. Phys. 140, 054113 (2014).

[19] A. Stanislavsky, K. Weron, and A. Weron, Anomalous diffusion approach to non-exponential relaxation in complex physical systems, Commun. Nonlinear Sci. Numer. Simul. 24, 117 (2015).

[20] M. Magdziarz, Langevin picture of subdiffusion with infinitely divisible waiting times, J. Stat. Phys. 135, 763 (2009).
[21] A. Stanislavsky, K. Weron, and A. Weron, Diffusion and relaxation controlled by tempered $\alpha$-stable processes, Phys. Rev. E 78, 051106 (2008).

[22] R. L. Schilling, R. Song, and Z. Vondraćek, Bernstein Functions: Theory and Applications (de Gruyter Studies, Berlin, 2010).

[23] S. Bochner, Diffusion equation and stochastic processes, Proc. Nat. Acad. Sci. USA 35, 368 (1949).

[24] A. Kumar and E. Nane, On the infinite divisibility of distributions of some inverse subordinators, Modern Stochastics 5, 509 (2018).

[25] N. Leonenko and E. Merzbach, Fractional Poisson fields, Methodol. Comput. Appl. Probab. 17, 155 (2015).

[26] F. Mainardi, G. Pagnini, and R. Gorenflo, Some aspects of fractional diffusion equations of single and distributed order, Appl. Math. Comput. 187, 295 (2007).

[27] C. H. Eab and S. C. Lim, Accelerating and retarding anomalous diffusion, J. Phys. A: Math. Theor. 45, 145001 (2012).

[28] M. Abramowitz and I. A. Stegun, Handbook of Mathematical Functions with Formulas, Graphs, and Mathematical Tables (Dover, New York, 1964).

[29] A. Stanislavsky and A. Weron, Transient anomalous diffusion with Prabhakar-type memory, J. Chem. Phys. 149, 044107 (2018).

[30] T. Sandev, W. Deng, and P. Xu, Models for characterizing the transition among anomalous diffusions with different diffusion exponents, J. Phys. A: Math. Theor. 51, 405002 (2018).

[31] T. R. Prabhakar, A singular equation with a generalized MittagLeffler function in the kernel, Yokohama. Math. J. 19, 7 (1971).

[32] R. Weron, On the Chambers-Mallows-Stuck method for simulating skewed stable random variables, Stat. Probab. Lett. 28, 165 (1996).

[33] A. Janicki and A. Weron, Simulation and Chaotic Behaviour of $\alpha$-Stable Stochastic Processes (Marcel Dekker, New York, 1994).

[34] A. Stanislavsky and K. Weron, Stochastic tools hidden behind the empirical dielectric relaxation laws, Rep. Prog. Phys. 80, 036001 (2017).

[35] A. K. Jonscher, Universal Relaxation Law (Chelsea Dielectrics Press, London, 1996). 